\title{
Ergänzendes Duplikat zu den Körperteilnamen SIL 122.
}

Vin II. Zimmern.

Zu dem von mir in dieser Zeitschrift Bd. 30 S. $288 \mathrm{ff}$. veröffentlichten Vokabular mit Körperteilnamen SII, 122 liegt, worauf mich WEIDNER aufmerksam machte, ein Duplikat vor in ciner Tafel aus Assur, die im Berliner Museum einstweilen allerdings nur durch eine Photographie (Nr. 4203) vertreten ist. ${ }^{x}$ Im $\Lambda$ nschlu $\beta$ an meinen früheren Artikel veröfentliche ich im Einverständnis mit der Direktion der Vorderasiatischen Abteilung und nach Übereinkunft mit WEIDNER, der mir auch seine $\mathrm{Abschrift}$ des $T$ extes freundlichst zur Verfügung gestellt hat, nach jener Photngraphie nunmehr auch dieses Duplikat, da es in seiner ersten Kolumne eine willkommene Ergänzung der dem Anfang von SIL 122 vorausgehenden Partie darstellt und in der zweiten Kolumne allerlei nicht uninteressante Varianten zu letzterem bietet.

Wie zu erwarten, bringt nun Kol. I von Photo 4203 (im folgenden $=$ C) solche Teile des menschlichen bzw. ticrischen Körpers, die, vom Kopf aus gerechnet, denjenigen in der erhaltenen unteren Ilälfte der Vorderseite von SII. 122

"Dic Tafel bot auf ihrer Rüclsscite jedenfalls den Schluls der Liste. Doch gibt die Photographic eben nur die Vorderseite wieder und es ist atus ihr auch nicht ersichtlich, ob das zu Ginude liegende (Originalfragment überhaupt dic beschriebene Rückseite enthält oder nur den Teil mit der Vorderseite $\mathbf{v}$ einer in der Mitte zwischen Vorder- und Rückseite durchgebrochenen Tafel, also eines sog. sandwich-tablet. 
(im folgenden $=\mathrm{A}$ ) vorausgehen, und zwar fehlt möglichrrweise überhaupt keine Zeile \%wischen C Kol. I und A Vorders. während der fehlende Anfang von $\mathrm{C}$, das vermutlich mit sag $=r \bar{e} s u$ «Kopf» begonnen hat, wahrscheinlich nur eine, die obere Kopfpartie behandelnde Abteilung enthielt, ron der noch die letzte Zeile, $[\mathrm{sag}-\mathrm{ki}=p u]-\hat{\imath}$-t $\iota$ «Stirn» erhalten ist. Durch solche Einteilung in Unterabteilungen durch Trennungsstriche unterscheidet sich überhaupt vorteilhaft $C$ von $A$, wie auch von $B$ (= Vok. Martin B), welch letzteres übrigens sicher ebenfalls aus Assur stammt.' I)as Fehlen solcher Trennungsstriche macht es - abgesehen von anderem $^{2}$ - übrigens auch unmöglich, woran man im ersten Augenblick wohl denken könnte, anzunehmen, daß B etwa zur gleichen Tontafel wie $C$ gehört und unmittelbar an dieses anschließt, obwohl durch einen neckischen Zufall $B$ genau an der Stelle abbricht, an der C einsetzt und mit fast genau derselben schräg nach unten verlaufenden Bruchlinie. Zur bequemeren Übersicht, zugleich auch in fugam vacui, füge ich in der Autographie auch eine Wiedergabe dieses Vok. Martin B bei, natürlich ohne jede Korrektur, in diplomatisch genauer Reproduktion der Autographie MARTIN's in Rec. de Trav. XXVII 125.

Auch diesmal wieder sehe ich, schon der Raumfrage wegen, von einer ausführlicheren Erörterung der einzelnen Körperteilnamen ab und begnüge mich mit einigen wenigen Bemerkungen in den Anmerkungen zu einzelnen Stellen. ${ }^{3}$

1) Da es «de même provenance» ist, wie Vok. Martin A, und letzteres, das "provient des environs de Mossoul» (jetzt in Berlin), sowohl laut Unterschrift wie aus sonstigen Gründen, Schriftcharakter usw., eine Assurtafel scin mul?.

2) Dahin gehört z. B. auch die verschiedene Art des Ausdrucks für das Wiederholungszeichen, in C durch $\mathrm{KI}-\mathrm{MIX}$ am Anfang der Spalte, in B durch zwei senkrechte Keile am Schluß der Spalte. Außerdem ist es unbedingt ausgeschlossen durch das Verhältnis von $\mathrm{C}$ Kol. II $;$ f. zu B Vs. 15-17.

3) $\mathrm{Zu}$ meiner früheren Veröffentlichung ron $\mathrm{A}$ sei hier nachtragsweise noch vermerkt, daß in Vs. 27 , in Übereinstimmung mit $B$, doch natürlich mi-lis-tum, nicht etwa mi-nu-tum zu lesen ist (der wagrechte Keil ist nur ein zufäliger Ritz im Ton, außerdem wird $n$ auf dieser Tafel ja ganz anders geschrichen). 
Dagegen möchte ich hier noch ctwas ausführlicher auf den schon in meinem ersten Artikel kurz berührten Zusammenhang unserer Liste mit der ähnlichen in CT XIV $3 \mathrm{f}$. und 5 $\mathrm{zu}$ sprechen kommen, da ich hier dank gütigen Mitteilungen EHELOLF's allerlei beizubringen vermag, was sowohl im einzelnen als im ganzen zur gegenseitigen Aufklärung zwischen diesen beiden Listen dienen kann. Dazu ist dann ferner neuerdings auch noch der engverwandte akkadisch-hethitische $^{\mathrm{I}}$ Vokabulartext mit Körperteilnamen KT Bogh. I Nr. 5 I (= Weidner, Studien S. 86 ff. Nr. 8, vgl. Delitzsch, Sum.akk.-hctt.Vok. S. 8) getreten. ${ }^{2}$

Das große 6-kolumnige Assurvokabular VAT I026I, von dem mir EHELOLF für die erste Kolumne freundlichst seine Abschrift des Originaltextes und für die übrigen Kolumnen eine kurze Inhaltsangabe zur Verfügung gestellt hat, ist in Kol. I Duplikat zu K. $4325+$ K. ${ }_{13692}$ (CT XIV 3 f.) Kol. I Z. 25 ff. und K. 4368 (CT XIV 5) Kol. I Z. I 4 ff., für die es allerlei wichtige Ergänzungen und Varianten, sowie insbesondere die unmittelbare Fortsetzung bringt, die dann auch die Verbindung mit K. 4396 (CT XIV 14) herstellt.

Ferner ist Rs. 23 statt $\mathfrak{u r} \cdot \mathrm{ku}$ nach $\mathrm{C}$ und nach VAT I026I besser $\mathrm{ib}-\mathrm{ku} z \mathrm{u}$ lesen, und Rs. 8 statt $s i-n i$ vielmehr $\$ i-t i k$ zu umschreiben. Endlich ist Rs. 27 nach VAT 1026I I 22 zu [uzuš̀g-se-se-g]a = par-su zu ergänzen und Z. 29 sicher zu [r]i-ki-tum (in Bogh. Nr. 5I Vs. 17 als ri-ik-ki-[du]; s. auch noch unten S. 19 Anm. 4 zu rigitu). Für Rs. $1-4$ ist jetzt auch PoEbel, HGT Nr. 137 zu vergleichen, wo u. a. dādānu mit Suff. I. Sing. als di-a-da-a-nu-ú-a erscheint.

1) Oder, wie man mit FORRFR jetzt vielmehr sagen soll, kanesische; vgl. dazu übrigens die Stadt Kanis CT XII 46, 33c, die man zeitweilig ja sogar in die Scheol versetzt hat.

2) Als Gegenstück hicrzu stoßen wir gerade in dem sumerisch-akkadischen Vokabular mit Körperteilnamen CT XIV 3. 5.14, mit dem sich Bogh. Nr. 5 I nahe berïhrt, anscheinend auch auf hethitische Wörter. So wohl sicher bei gu-ru-us-bi-ra-as, dem letzten der vier Synonyma von bișsuru "weibliche Scham" (das guristu CT XIX 40, 2ob, das ToRCzNer, ZDMG 66, ; i l wohl mit Recht dazu stellt, wird dann die semitisierte Form des hethitischen Wortes sein), so wohl auch in den merkwürdigen beiden Wörtern für "Tiermagen» kukudur bzw. kukkadru, kukudru und misissam (s. dazu unten zu VAT10261 Kol. I'33):

Zeitschr. f. Assyriologie, XXXIII. 
Kol. I von VAT io26I lautet folgendermaßen: (1) [uzu ] $[],(2)\left[\begin{array}{ll|l|l}\mathrm{uzu} & \mathrm{ni} & \check{s} \epsilon-e-m u & s i-[m e-t \hat{u}]\end{array}\right]$

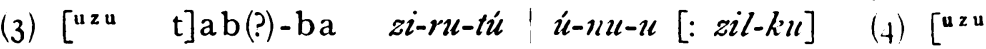
1]íl-1á $|m u n-d u-l u| s i-i-r u$ pu-ut-t[u-u] (5) [uzu m]in-tab-

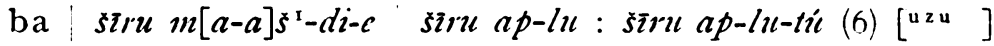
gi-keš-da $|g a-g u-r i-t u| b a-m u-r i-t u ́ c(7)[u z u]$ ùb(?) $\mid m a-$

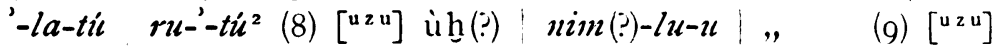
eme | ma-'-la-tú |li-ša-a-mu (1о) [uzu] síl(う)-gal ma-la-ku (I I) [uzugag(?)] ti-ti | ba-ma-tú sik-kat si-li (12) [uzugag t] i-tur na-as-pa-du si-ti-ik ir-ti (13) [uzugag-

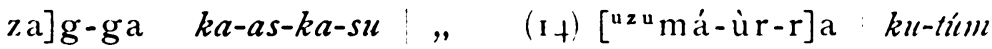
$l i b-b i \quad b i-i n-s u\left({ }_{15}\right)[\mathrm{uzu}]$ šàg-gig : sí-qu tu-li-mu.

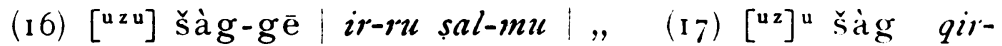

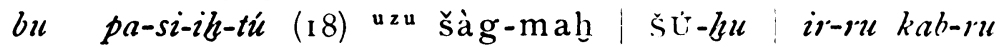

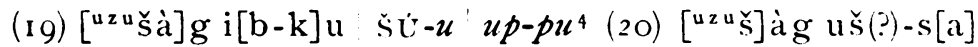
$\check{s} \dot{u}-u \mid, \quad(2 \mathrm{I})[\mathrm{uzu}]$ šàg-šú-nigin $i r-r u$ sa-bi-rnt-[t]u|ti$r a-n u^{5}(22)\left[{ }^{u}\right]^{z u}$ šàg-se-se-ki $|p a r-s u|$ "sin-ni (23) [u $]^{\mathrm{zu}}$ šàg-gar-gar-ra | su-ru-um-mu ir-ru ga-mir-tú (24) ["] $]^{z u}$ šàg lib-bu|,, , (25) [uzu] š[à]g-[ ]gar $\mid m e-i-r u^{6} \quad u-$

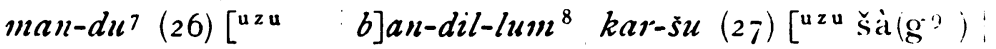
$\left.p\left(a-p a-a n l i b b i(?)^{\mathrm{ro}}\right)\right] \quad, \quad(28)^{\text {uzu }}$ šàg-[. (.) . ba(n-tíi)] (29) [u] u maš-k[un kin-(na-tú)|, , " " (30) [u]zu nam-gar

I) Var. [m]as. Dieses masdū mit Ideogr. min-tab-ba ist wichtig für Enuma eliš IV 137! Danach wohl auch meine Ausführungen dazu OL.7. 191\%, 104 z.1 modifizieren.

2) Var. $u r 0^{\prime}-\dot{u}-d u$; so auch in Bogh. Nr. 5 I Vs. 2 f. Vgl. Hor.M. $43 \mathrm{Anm}$. I.

3) In Bogh. Nr. 51 Vs. 13 als du-li-im-mu.

4) Vgl. hierzu auch CT XVII 25, 27: [ib]-ku=up-pa a-hi. Darnach SAI 7970 zu verbessern. 5) In Bogh. Nr. 5 I Vs. I I als te-ra-a-mu.

6) Ebend. I 2 als me-ir-du; auf A Rs. 16 als mi-i-ri (=uzu (mu-ru) $m ! l r$ ).

7) Oder sam-man-du? Auch VAT 8917 Vs. 6 unter Körpertcilen.

8) Vgl. ban-dil-lu $=[$ kar $]-s_{u}^{\prime}$ in VAT $10613 \mathrm{Rs}$.

9) Von hier ab mit Ergänzungen nach K. 13602 (C) $\mathrm{XIX}$ 13), vgl. Meissier, MVAG 1904, 249 und Holma 172.

10) Diese Ergänzung von mir nach der Folge bäntu, papän libbi, kinnatu CT XXVII 34, 20 ff., vgl. Holma 72.

i1) Von hicr ab mit Ergäinzungen nach K. 4396 und alsbald auch Sm. 995 (beide CT XIV I4). In der Ausgabe von Sm. $995 \mathrm{~V} \mathrm{R}_{27} \mathrm{Nr} 3$ (Pixches) 


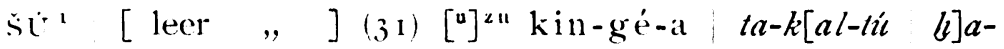
$\check{s}[u]-u^{2}$ (32) [u] $]^{z u} \mathrm{kin}-\mathrm{gé}-\mathrm{a} ; a-m u-\left[t u \mid g a-b i-d u^{3}\right]$ (33) $\left[{ }^{\mathrm{u}}\right]^{\mathrm{zu}} \mathrm{kun}$-šàg-ga $\mid$ šar-q[a-tú |ku-ku-díu : meš-ès-àm $\left.{ }^{4}\right]$ (34) $\left.{ }^{\mathrm{u}}\right]^{\mathrm{z} u} \mathrm{kun-šàg-ga} \mid k u-k u-b a-t u ́: p i-i[k a r-s ̌ i \mid k u-k u-$

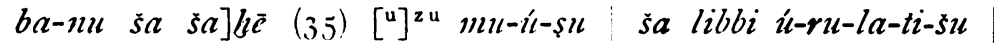
[pap-pal-tu sa bir-ki améli] (36) ["i] bar zabar I nam"gàl-lu aban bi[r-ki améli]. Darauf Trennungsstrich und noch 4 Zeilen mit Stcinnamen, die aber nicht mehr mit K. 4396 und Sm. 995 (beide CT XIV 14) übereinstimmen (vielmehr $u s ̌ n, ~ u k n u \grave{u}$ usw. behandeln). Aus dem Vorstehenden folgt also, daß K. 4396 und Sm. 99.5 zum gleichen Text wic I. 4325 (CT XIV ${ }_{4}$ f.) und K. 4368 (CT XIV 5) gehören. Auf die Namen von Körperteilen - darunter gegen Ende dic beiden für die Wahrsagekunst ja besonders bezeichnenden Wörter takaltu und amütu - folgten demnach (und dies wird auch durch das große Assurduplikat VAT I026 I im

wie auch noch CT XIV I4 (THOMPSON) sind Vs. und Rs. verwechselt, während BEzold in Catalogue unter Sm. 995 bereits das Richtige bot.

I) d. i. namgarru; so gewiß aufzufassen wegen VAT I06I3 Rs. namsar-ru $=$ [,] (d. i. [kar]-su). Darnach SAI 125; zu ändern.

2) Als $h a-s i \dot{u}(!)-i i$ in Bogh. Nr. $5 \mathrm{I}$ Vs. 6.

3) Ebend. Z. 9 als $g a-b i-d u$. Auch K. 4396 ist sicher $g[a]-b i-d[u]$ zu ergänzen, nicht $q[i] r-b i-t[u m]$ HoLsi 69; ebenso 93040 (CT XII 2 I) Rs. I 8 a [g]a-bi-due (gegen MeIssner, SAl 6420; Hoima $75^{1}$ ), während die Parallele Br. 8531 allerdings $k a-b i-d u$ bietet. ta-kal-tú $=g a-b i-d u$ auch in VAT $9 ;$ I 8 vs. Kol. III, dagegen in VAT 10613 Rs. 55 f. $k a-b i-t u=k a-b i-d u, a-m u-t u=$, .

4) VAT 10613 Rs. 57-59: sar-ga-tu =ku-uk-kad-ru; ku-uk-kad-ru= $r i$-ki(?)-tii (s. dazu oben S. 17 Anm.);,$=m i$-sis-sa-am. In Bogh. Nr. 51

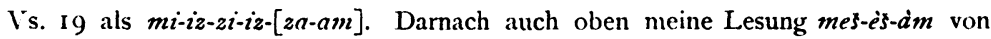
mes-AN-A-AN. Zu diesem ku-ku-dier, ku-uk-kad-ru (wofür aber wohl besser ku-uk-kiud-ru zu lesen ist) gehört ferner ku-ku-zd-ru (also nicht kukubarru zu lesen!) in 80-7-19,80 (= Bolssier, DA 97) und in K. 4112 (s. Bezolo, $\mathrm{Cr}$ talogue sub Nr. und BoIssif.r, Choix I I08). Darnach auch Holma I4; Anm. 2 zu ändern. \%ur eventuellen Herkunft dieser Wörter aus dem Hethi-

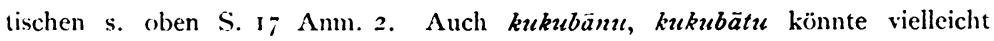
ursprünglich aus dem Hethitischen stammęn und in seinem ersten Bestandteile dasselbe Element enthalten wie kukudur, kukudru. Die aramäischen Formen קורקבניא usw. beruhen ja wohl sicher erst auf Entlehnung aus dem Akkadischen (vgl. AF 48). 
cinzelnen noch des Weiteren bestätigt) zunächst Stcinnamen. darunter an erster Stelle magische, zu Beschwörungszwe'cken verwendete Steine; sodann Pflanzennamen (beginnend K. $4 ; 25$ Rs. rechts Z. 2); darauf Fischnamen (Schluß derselben in Sm. 995 Rs.(!) Z. 2); darauf Vögelnamen (beginnend cbenda Z. 3 , sich fortsetzend in K. $8202+$ Sm. 1438 (CT XIV 5) und weiter in K. 4325 Rs. links bzw. K. 4368 Rs.). Die letzte, sechste, Kolumne des Assurduplikats enthält endlich noch Namen von Gewändern usw. In der AssurbanipalBibliothek entspricht vielleicht, mit EHELoLf, K. 42 I I CT XVIII I7), das ja auch vermutlich eine (fast gänzlich abgebrochene) sumerische und sodann zwei entsprechende akkadische Kolumnen aufweist.

Aus der nahen Verwandtschaft zwischen SII, 122 und seinen Duplikaten mit dem im vorstehenden besprochenen großen Texte mit Namen für Körperteile, Steine, Pflanzen, Fische, Vögel, Gewänder dürfte sich, wie ich schon in meinem ersten Artikel vermutet habe, von neuem mit ziemlicher Sicherheit ergeben, daß es sich bei jener Liste mit Körperteilen im wesentlichen um Teile des Tierkörpers, genauer wohl noch des Schafes, handelt und zwar in ihrer Beziehung zur Wahrsagekúnst, speziell Opferschau, und Beschwörungskunst. Vielleicht ist es auch kein Zufall, daß unter den Tierkörperteilnamen dieser Listen gerade auch einige hethitische Bezeichnungen zu begegnen scheinen (s. oben S. 19 Anm. 4). Es könnte dies, im Zusammenhang mit den in Boghazköi gefundenen Schafslebermodellen und anderem, auf einen besonders engen Zusammenhang zwischen babylonischer und hethitischer Eingeweideschau hinweisen, wie andrerseits ja, durch die Etrusker vermittelt, diese besondere Art der Wahrsagekunst von Kleinasien aus nach Rom gekommen sein wird.

Bei dem im folgenden vorgelegten Assurduplikat zu SIL 122 ist noch besonders zu beachten, daß dieser Text, wie so manche Assurtexte, offenbar von einem nicht sehr sorgfältigen und vielleicht auch nicht besonders unterrichteten Schreiber niedergeschrieben ist, man daher mit direkten 
Schreibfehlern rechnen mul3. SIL I 22 bictet im Zweifelsfalle entschieden die bessere Lesart.

\section{Kolumne I.}

(Das Vorhergehende abgebrochen.)

\begin{tabular}{|c|c|c|c|}
\hline [uzu sag- & $\mathrm{ki}$ & $p u]-$ & $t u$ \\
\hline$[\mathrm{uzu}$ & ].$^{1}$ & $i-$ & $s u^{2}$ \\
\hline [uzu $].-$ & gal & $l a-a \check{s}-$ & $b u^{3}$ \\
\hline 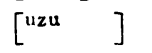 & gal & $l a-b u-$ & $\dot{u}^{4}$ \\
\hline$\left[\begin{array}{l}\text { uzu } \\
\end{array}\right.$ & gal & $k a-a n-z u-$ & $z u^{5}$ \\
\hline$[\mathrm{uzu}]$ & și & mar - & $t u^{6}$ \\
\hline$[\mathrm{uz}]^{\mathrm{u}}$ & gú-bar & S̀U - $u$ & \\
\hline$[\mathrm{uz}]^{\mathrm{u}}$ & țu-bar ${ }^{7}$ & $s a-a p-s a-$ & $p u$ \\
\hline
\end{tabular}

1) Ergänzung zu [me-s] i erscheint nicht möglich, da auf der Photographie am Schlusse deutlich nur ein senkrechter Keil zu sehen ist.

2) Vgl. zu isu, issu "Kinnbacke» (sum. me-și) außer Holma 33 f. noch denselben, ZA 28, I6I (nach LANDSBerger) und vorher schon Jenses, ZDMG 67, 509 (und in brieflicher Mitteilung an mich vom 5. II. '08: ReISNER, Hymn. Nr. 79, 10 is-si-šu (= me-și-bi) $\dot{u}$-si-id «seine Backe machte er erröten»); gewiß mit JeNsex, a. a. O. auch in dem (meist phonetisch geschriebenen) Gestirnnamen (Stier, Hyaden) is le «Kinnbacke des Stiers», II R 49, 45 dagegen ideographisch mulgud-an-na $=$ uzume-și(!) li-e = la-bi-e al-pi (!).

3) lašlu liegt, außer an den von Holma 24 angeführten Stellen, nur mit Lautumstellung, auch an der bekannten Assurbanipal-Stelle Rm. Zyl. IX 107 vor, wo natürlich la-ab-si-su zu lesen ist. So richtig auch bereits BoIssier, PSBA 20 (1898), 163, sowie Jelitto, Diss. 57, wohl im Anschluß an MeissNer, s. letzteren MVAG I904, 223 Anm. 5. STreck dagegen liest noch la-ah päni-su. Aus diesem Nebeneinander von lašbu und lab̧su erklärt sich auch sehr einfach, als eine Kontamination des Schreibers, das la-as-bi-si-im in CT XVII 50, I8. la-as-hu, neben anden Körperteilen, auch VAT 891 ; Vs. 8.

4) lah̆ü liegt wohl auch vor KING, Mag. Nr. 6I, 18: ina la-ḩi-ka sūusäa.

5) kanzuzu doch sicher nur eine Nebenform zu kusäsu («Zahnwurzel»?), das ja in der "Zahnwurmbeschwörung" gleichfalls in Verbindung mit lashu genannt wird.

6) martu hier natürlich nicht in Sinne von «Galle"; dagegen dürfte an imat marti Utuk. lemn. Taf. I, Kol. I $16 / 1 ; \%$ erinnern sein.

7) So, !u-bar, ist sicher au tesen, nicht $k u-b a r$, wie WEIDNe, OLZ Iy12, 20y an unserer Stelle liest. Durch dieses Ideogramm wird demnach auch, 


\begin{tabular}{|c|c|c|c|c|}
\hline & {$[\mathrm{uzu}]$} & $\mathrm{ka}$ & $a p-$ & $p u$ \\
\hline 10 & {$[\mathrm{uzu}]$} & $\mathrm{ka}^{\mathrm{I}}$ & $\check{s} i-i n-$ & $m u$ \\
\hline & {$[11 z u]$} & ka & $p u-$ & $\dot{u}$ \\
\hline & {$[\mathrm{u}<\mathrm{u}]$} & murub 2 & KI-NIN & \\
\hline & {$[\mathrm{uzu}]$} & unu & KI-NIN & \\
\hline & {$[\mathrm{u} z \mathrm{u}]$} & un & KI-XIN & \\
\hline 15 & {$[\mathrm{uzu}]$} & $\mathrm{emc}$ & $l i-s \dot{a}-a-$ & $n u$ \\
\hline & {$[u<u]$} & eme & mal(3) ${ }^{3}-$ & ' $u$ \\
\hline & {$[u z]^{u}$} & eme & $\operatorname{mal}(?)^{3}-^{3} a-$ & $t u$ \\
\hline & {$[\mathrm{uz}]^{\mathrm{u}}$} & emc & $s a p-$ & $t u$ \\
\hline & {$[\mathrm{uz}]^{\mathrm{u}}$} & $a-\stackrel{u}{ }$ & $a-m u-$ & $i$ \\
\hline 20 & [uzu & $a-\stackrel{u}{ }$ & $a-l i q \quad p i-$ & $e^{\prime}$ \\
\hline
\end{tabular}

entyegen den Ausführungen WeIDNER's daselbst, der für sapsapu die Bedeutung aGurgel» erschließen wollte, für dieses vielmehr die Bedeutung "Unterlippe: an die Hand gegeben, da ta-bar bekanntlich ja (so auch in der ideographischen Schreibung des Namens Gilgameš) $=$ saptu saplitu Unterlippe, ist. C̈ber das für das altassyrische Strafverfahren charakteristische "Abschneiden der Unterlippe» bald an einem andern Orte!

I) Bzw. in dieser Bedeutung sú zu lesen.

2) Doch sicher das Zeichen Br. 10959. Vgl. für diese Zeilen die gleiche Gruppe in CT XII $36,1-3=38,1-3$ und dazu Delitzsch, Sum. Gloss. 53 unter un u II und S. I92 unter muru(b) I.

3) Oder ma-'u, ma-'a-tu? In diesem Falle wäre dann aber doch wohl zugleich eine Verschreibung für mal-'u, mal-'a-tu oder für ma-al-'u, ma-al'a-tu anzunehmen. Vgl. oben VAT $1026 \mathrm{I}$ I 7.9 ma'latu (das dann eine Umstcllung von mal'atu wäre) $=r \iota^{\prime} t u$ (bzw. ur'udu) und $=l i s a \bar{n} u$ und für einen Stamm $l a^{\prime} u$ "schlürfen", =לעy und CT XVIII 30, 2 I it-ti-la-" (= $\mathrm{NAG}_{\text {) }}$ in einer Gruppe mit sikru, sikru satī und läsu (vgl. dazu HAUPT,

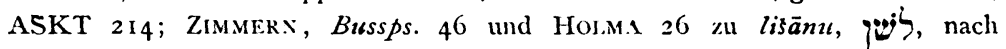
HAUPT und BITTNER) mit gleichem Ideogramm, ferner auch noch (mit Ehelof.F) CT XVIII 21 , Sin. 1744 la- $-u$ neben $r \iota^{\circ}[u \iota]$, und vielleicht doch auch $u \iota^{\circ} u$ (s. Holma 3 I), das dann allerdings nicht "Backe", sondern eher etwas zur Zunge. Gehöriges bezeichnen würde.

4) Dazu la-aq pi mit dem gleichen Idengramm, s. Holm. 24 f., des-

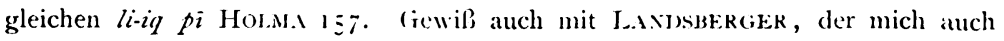
noch auf $l a-a q p i-s u=s a-m e-e$ pi-su bei PINchls, JRAS I9 12, 833 hinweist, KiNG, Mag. Nr. 53, 10 lig pi-ja ubbalu ameinen Gaumen lassen sie vertrocknen» zu lesen; ebenso mit demselben mubbil liq $p[i-i]$ KTAR Nr. 88 Fragm. 4 Vs. rechts 4 ; vgl. KTAR Nr. So Rs. 28 (Ekrisci, Quellen 130 ) in ganz :ilhnlichem Zusammenhange: ru'ti ubbilu. 


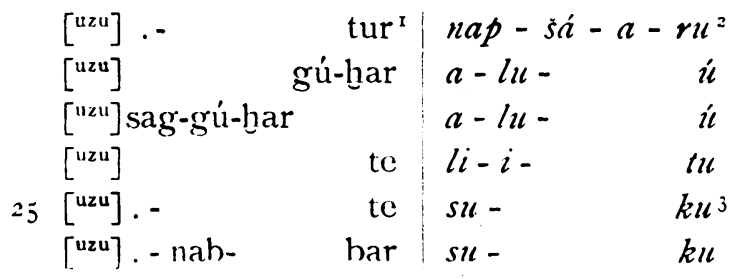

(Fortsetzung abgebrochen.)

\section{Kolumne II. ${ }^{4}$}

(Das Vorhergehende abgebrochen.)

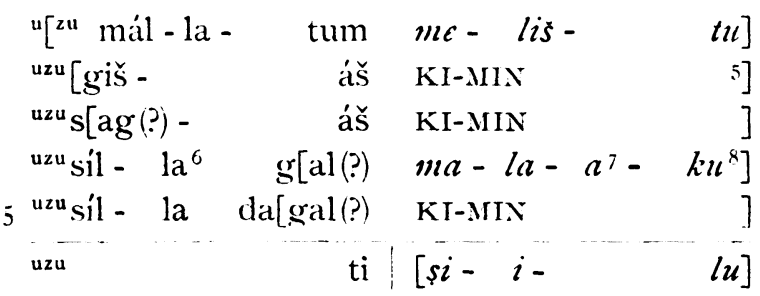

1) Eine Ergänzung etwa zu uzu a- $\mathrm{u}$ tur ist den Raumverhältnissen nach unmöglich, wenn auch die beiden senkrechten Keile an der Bruchstelle um eine Kleinigkeit weiter nach rechts stehen, als es meine Autographie bictet.

2) Dieses napsäaru bezeichnet natürlich einen andern Körperteil als das napiarn auf Rs. 14 .

3) Natürlich identisch mit dem von HOLM. 150 f. behandelten usukku, das ja ebenfalls gerade auch neben lètu erscheint. Im Hinblick auf sein te enthaltendes Ideogramm wird daher auch suku, usukku etwas dem Begriff Backe: Nahestehendes bezeichnen.

4) Hier gebe ich nicht alle Varianten von A (und B), sondern nur die wichtigreren.

5) Die Ergänzungen in Z. $2-5$ sind nicht ganz sicher; wie es scheint, fehlt in C zwischen Z. 3 und + uzue-is $=K I-M I x$ gegenüber $A$ und $B$ : andererseits bietet es in $\%, 5$ anscheinend ein Plus gegenüber $A$ und $B$.

o) -la fehlt in A.

8) Durch maläku in V.AT 1026 I I Io (s. oben S. 18) wird sowobl die Lesung maläku gegenüber M.রTIN's malälu als richtig erwiesen, als auch dic Bedeutung "Zunge" für malaku festgelegt (nach EHeloly von ك) lecken:). Natïrlich handelt es sich aber hicr nicht um die eigentliche Junge im Munde, sondern un einen mit \%unge (eig. Lecker benannten kïrperteil in der Weichengegrend. 


\begin{tabular}{|c|c|c|c|c|}
\hline & uzu ti - & $\mathrm{ti}$ & $\mathrm{KI}-\mathrm{M}[\mathrm{IN}$ & ז] \\
\hline & vzu ti - & $\mathrm{ti}$ & $b a-a[n-$ & $\left.t u^{2}\right]$ \\
\hline & uzu gag - & $\mathrm{ti}$ & sik-kdt [si- & $l i]$ \\
\hline 10 & uzu gag - zag - & ga & $m \grave{a}(\grave{0})^{4}-k i-.[$ & $5]$ \\
\hline & uzu gag $^{6}-$ ter - & $\mathrm{ra}$ & $b a l^{7}-t a p-[p a-a-$ & $t u]$ \\
\hline & uzu & gab & ir - & $t u]$ \\
\hline & uzu $\operatorname{tar}^{8}-$ & gab & $s i-d i-$ & $,]$, \\
\hline & uzu áb - & gab & $s i r^{10} n a p-s[\dot{a}-$ & ri] \\
\hline J & uzu áb - & ter & $m \dot{a}(\stackrel{)}{)}-$ & $\left.{ }^{\prime} u^{11}\right]$ \\
\hline
\end{tabular}

1) Diese Zeile fehlt in $A$.

2) A ba-ma-a-ti, B ba-a-ma-tu und wahrscheinlich in der folgenden Zeile als ein Plus gegenüber $A$ und $C$ noch $\left[\mathrm{u}^{2} \mathrm{u}_{\mathrm{ti}-\mathrm{ti}}=b a\right]-a n-t u$. Desgleichen bietet VAT 1026I I I [ [uzu]ti-ti $=b a-m a-t i ́ b=s i k$-kát si-li, sowie Bogh. Nr. 5 I Vs. 16 ba-an-du. Auch Yale Syll. 243 liegt gewiß [ba-ar! BAK] ma-a-šu |ba-an-tum vor (vgl. das Faksimile, wonach ba wohl über Korrektur geschrieben). Darnach wohl auch CT XII 16, 58 b (die Stelle feblt SAI S. 50) ba-ár|BAR ba-rum|ba-a[m-tuc] (so hier nach den Spuren, auch bei MrissNER, Suppl. S. 28 der Autographien, wohl besser als $b a-a[n-t u c]$ zu erginzen; cbenda Z. 60, 6I (s. SAI Io65) übrigens $s a ́$-[am-tu], nach Yale Syll. 24i, wo statt $\ddot{u}$ $z u m r u$ und kabittu und steckt auch in bar-sil neben sil = naglabu. Damit behält HolmA 55 ff. in der Hauptsache Recht gegenüber WEIDNF, OLL 1914, 496 f., der überdies die Ausführungen HolmA's mehrfach mißverstanden hat.

3) A noch: uzugag-ti-tur = na-as-pa-du (vgl. dazu HoLMı, OLZ I9I4, 496, aber auch Christias, ebend. 396), das in VAT 10261 I 12 weiter noch $s i-t i-i k$ ir-ti gleichgesetzt wird.

4) Dieses Zeichen, das noch in Z. 15, 16, 1\%, 30 begegnet, kann doch wohl nur das Zeichen dir, mal mit dem Lautwert má sein.

5) A kas-ka-su; VAT $1026 \mathrm{I}$ I,I 3 ka-as-ka-su und = si-ti-ik ir-ti.

6) A noch -zag-ga. 7) A $k a$; obiges hal also wohl nur Schreibfehler.

8) A giš.

9) A si-tik (für den Lautwert tik des Zeichens vi s. HILPRFCHT, Assyriacu 26 Anm., Peiser, OLZ igO4, 9).

ro) A si-i-ri.

I) Statt Z. 15-1; bietet A uzuab-ter $=p a r-s u$, uzuab-dun $==,$, , uzuli-dur $=a-b u-u n-n a-t u m$; V.IT $1026 \mathrm{I}$, an einer allerdings erst ctwas späteren Stelle, 1 22: u«usàg-se-se-ki $=$ par-su $=$, (d. i. ti-ra-nuc) sin-ni. ... $m \grave{a}^{\prime} u$, die richtige Lesung und Ergänzung vorausgesetzt, wäre dann natïrlich $=$ usw. Eingeweide . Dieses $m a 0^{*} u$ "Eingeweide» liegt, wie ich glaubc nachgewiesen zu haben (s. MVAfi 1916, 218 Anm. 1), auch Enuma clis 1101 vor. Ob dazu am Ende auch dats von Holas 94 besprochene M. gehörti 


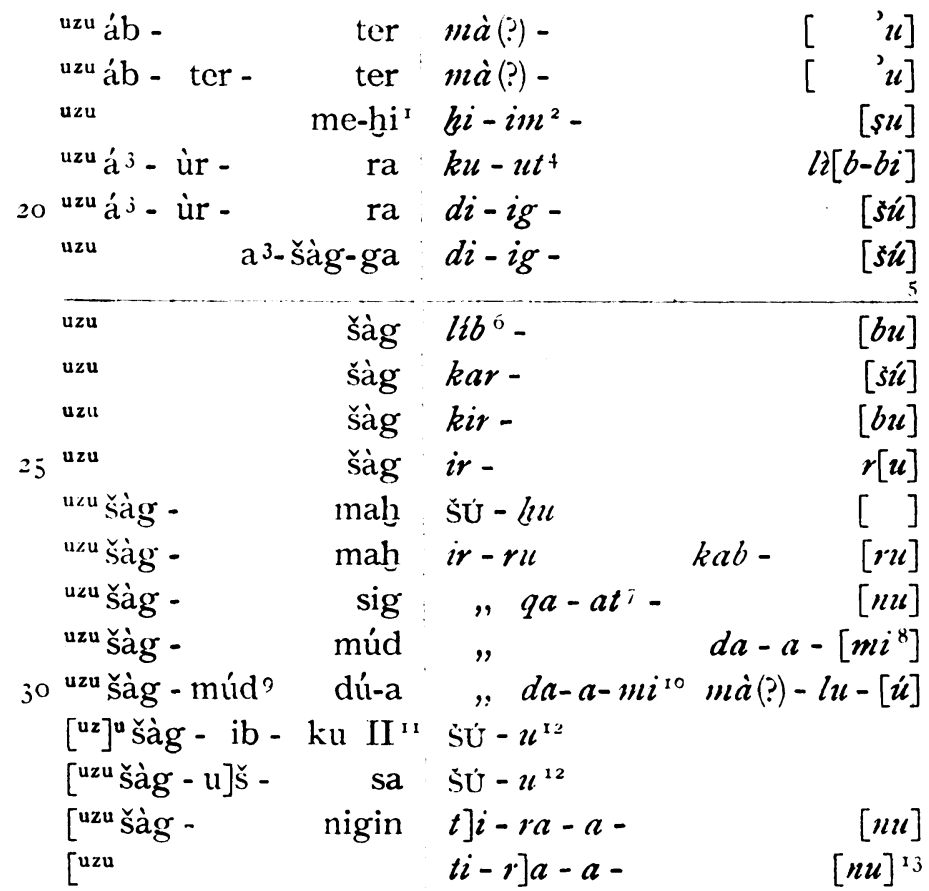

(Fortsetzung abgebrochen.)
I) A hà ; obiges hi wohl nur Schreibfehler.
2) A in.
j) A má.

4) A túm; ebenso VAT $1026 \mathrm{II}$ I4 und: ku-túm lib-bi $=h i-i n-\$ u$; Bogh. Nr. 5 I Vs. 8 ku-ut-mu libbi. Darnach $k u t-u t$ oben gewiß nur Schreibfehler für ku-ut-mu. Die Stelle lehrt ferner auch durch die Form himsu und durch die Gleichsetzung mit kutum libbi, daß hinşu nicht mehr $=$ חלצים usw. gesetzt werden darf, sondern vielmehr jüd.-aram. חופצא *Fettschicht auf dem Magen" entspricht. Dazu wird dann auch das hinsu, bimsu bei Holms 62 Anm. 2 gehören, das Jensen, KB VI 2, I S. 3 Z. 13, wohl ebenfalls im Hinblick auf חומצא, mit «Talg: übersetzt.

5) A noch die Zeile: uzu mu-rumur $=$ mi-i-ri; VAT I026I 125 $[\mathrm{u} \% \mathrm{u}] \mathrm{s}[\mathrm{i}] \mathrm{g}(\because)[] \cdot \operatorname{gar}=$ me-i-ru $=\dot{u}-\operatorname{man}-d u$; Bogh. Nr. $5 \mathrm{I}$ Vs. 12 als me-ir-du.
6) A lib.
7) A qat.
8 ; Dicse Zeile fehlt in A.
9) A mud.
10) A $s a d a-m u$.

11) Oder ib-ku-a: Aber nach Photographic kaum möglich. Schreib-

fehler: $\mathrm{A}$ blok ib(!)-ku.

(a) In VAT 1026 I I Ivf. mit up-pu gleichgeset/t.

ij) Diese Zeile anscheinend cin Plus gegenüber $A$. 


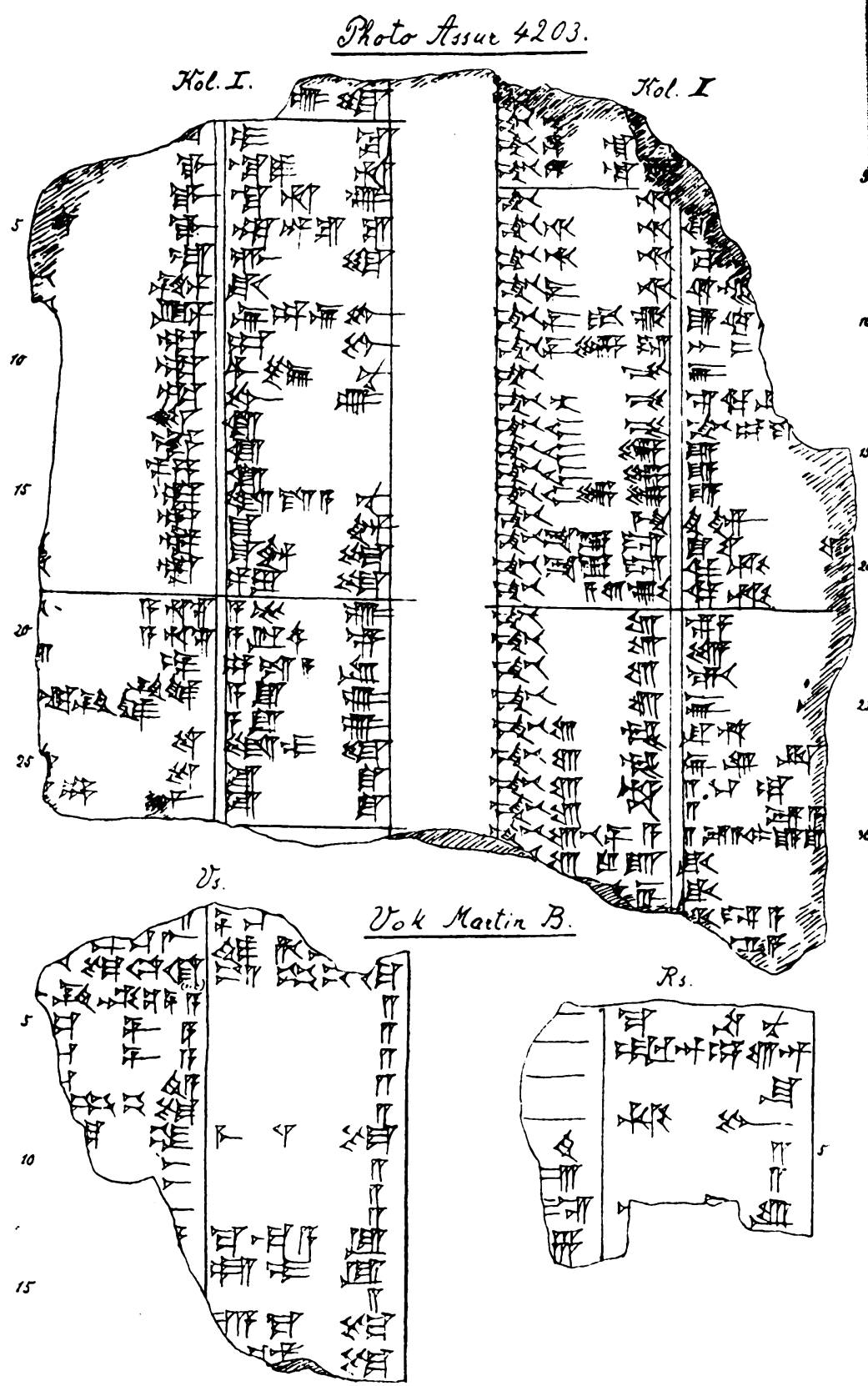

